\title{
Peripheral Differential Leukocyte Counts in Humans Vary with Hyperlipidemia, Smoking, and Body Mass Index
}

\author{
Zei-Shung Huang ${ }^{a}, *$, Kuo-Liong Chien ${ }^{a}$, Chi-Yu Yang ${ }^{a}$, Keh-Sung Tsai ${ }^{b}$, and Chiu-Hwa Wang ${ }^{b}$ \\ Departments of ${ }^{a}$ Internal Medicine and ${ }^{b}$ Laboratory Medicine, College of Medicine, \\ National Taiwan University, 10016, Taipei, Taiwan, Republic of China
}

\begin{abstract}
Reports of diverse relationships between blood concentrations of different lipids and peripheral total leukocyte count, and a unique lower peripheral monocyte count in hypercholesterolemia, have driven us to think that in humans, peripheral differential leukocyte counts may be influenced differently by different types of hyperlipidemia. Our subjects were Taipei residents who attended a regular health check program in our hospital in 1998. A total of 3,282 subjects was enrolled, including 1,677 normolipidemic, 960 untreated borderline hyperlipidemic, and 645 untreated hyperlipidemic subjects. By one-way analysis of variance (ANOVA), we found that different types of hyperlipidemia were associated with significant differences in differential leukocyte counts. In hypertriglyceridemia, the total leukocyte count and counts of all leukocyte subtypes were significantly higher than those in normolipidemia. Pure hypercholesterolemia, by contrast, was associated with a significantly lower monocyte count and no significant difference in other leukocyte counts. By two-way ANOVA adjusted for presence and degree of hyperlipidemia, we found significantly higher counts of total leukocytes and of all leukocyte subtypes in smokers, and significantly positive trends in relationships between body mass index (BMI) and counts of all leukocytes, neutrophils, lymphocytes, and monocytes. By multivariate regression analysis including all subjects, the serum triglyceride (TG) level was positively correlated with total leukocyte count and counts of all subtypes except eosinophils. On the contrary, serum high density lipoprotein-cholesterol had a negative correlation with total leukocyte count and with counts of neutrophils, monocytes, and basophils. In these multivariate regression analyses, there was no significant correlation between lipid levels and eosinophil count, whereas smoking was consistently associated with significantly higher counts of all leukocyte subtypes, including eosinophils. BMI had a significantly positive correlation with counts of all leukocytes, neutrophils, lymphocytes, and monocytes.
\end{abstract}

Paper no. L8472 in Lipids 36, 237-245 (March 2001).

Reports of a positive association between human peripheral total leukocyte counts and serum triglyceride (TG) levels $(1,2)$, a negative association between peripheral total leuko-

*To whom correspondence should be addressed at Department of Internal Medicine, National Taiwan University Hospital, No. 7, Chung-Shan South Rd., 10016, Taipei, Taiwan, R.O.C. E-mail: barrat@tpts7.seed.net.tw

Abbreviations: ANOVA, analysis of variance; BMI, body mass index; CVD, cardiovascular diseases; HDL, high density lipoprotein; LDL, low density lipoprotein; SE, standard error; TC, total cholesterol; TG, triglyceride. cyte count and serum high density lipoprotein (HDL)-cholesterol level (1-3), and a lower peripheral monocyte count in hypercholesterolemia (4) suggest that different types of hyperlipidemia may influence peripheral differential leukocyte counts differently. There is little information concerning this issue so far in the literature. An in-depth study on this point may help in understanding the pathophysiology of hyperlipidemia-induced atherosclerosis, since accumulating evidence from human studies has shown that hyperlipidemia is a major cause of atherosclerosis and cardiovascular disease (CVD) (5-7), that different types of leukocytes are likely to play important but different roles in atherosclerosis (8-10), and that a higher peripheral total leukocyte count is associated with an increased incidence of CVD (3,11-13). In addition, because cigarette smoking and obesity are frequently found in association with both lipid disorders (14-16) and a higher peripheral leukocyte count $(1,3,13,17-19)$, we included the factors of smoking and body mass index (BMI) in the study.

\section{MATERIALS AND METHODS}

Study subjects. Our subjects were selected from participants of a regular health check program in our hospital in 1998. The first condition for enrollment was to be a resident of the Taipei area including Taipei City and neighboring Taipei County. This condition was to ensure the feasibility of telephone interviews of hyperlipidemic and borderline hyperlipidemic subjects to see if they had received medication for hyperlipidemia before undergoing their health check in 1998. Among Taipei residents in 1998 who attended our health check program, 4,461 subjects were selected initially because they fit the following two conditions: (i) they had received serum lipid analysis with the same biochemical analyzer (Automatic Multichannel Biochemical Analyzer, Hitachi-7450; Hitachi, Tokyo, Japan); and (ii) they had received peripheral leukocyte analysis with the same blood cell counter (Sysmex Cell Counter NE-8000; TOA Medical Electronics, Kobe, Japan). Among the 4,461 subjects, 67 cases with serum TG level $\geq$ $450 \mathrm{mg} / \mathrm{dL}$ were separated as a special group because of the profound influence of their very high TG levels on the laboratory measurements of serum low density lipoprotein (LDL)cholesterol levels. In the remaining 4,394 subjects, diagnoses of normolipidemia, untreated hyperlipidemia, untreated bor- 
derline hyperlipidemia, and others were made according to the criteria listed in the following Diagnostic criteria for hyperlipidemia subsection, based on health records and results of telephone interviews for the history of and treatment for hyperlipidemia. Telephone interviews were conducted as follows. For each subject, at least seven calls were made, each call on a separate day, with the aim of having verbal communication with the enrolled subject personally over the telephone. Information from the family was considered unreliable and was not taken. Only information taken from the enrolled subjects themselves was used to conclude whether they had received medication for hyperlipidemia before undergoing the health check in 1998. Ultimately, 3,282 subjects were enrolled in the study, including 1,677 normolipidemic subjects, 645 untreated hyperlipidemic subjects, and 960 untreated borderline hyperlipidemic subjects. The 645 untreated hyperlipidemic subjects were subclassified into 102 cases of pure hypercholesterolemia, 187 cases of pure hypertriglyceridemia, 111 cases of combined hyperlipidemia, and 245 cases of probable combined hyperlipidemia.

Laboratory data. The following data were collected from the health records: (i) age, gender, weight, height, existence of a smoking habit, and history of and treatment for hyperlipidemia; and (ii) results of serum lipid study and leukocyte analysis. BMI was calculated as body weight/body height ${ }^{2}$ (in $\mathrm{kg} / \mathrm{m}^{2}$ ). The items of the lipid study included serum levels of total cholesterol (TC), HDL-cholesterol, LDL-cholesterol, and TG (all in $\mathrm{mg} / \mathrm{dL}$ ). The leukocyte analyses included total leukocyte count (in $10^{9} / \mathrm{L}$ ) and differential percentages (\%) of neutrophils, lymphocytes, monocytes, eosinophils, and basophils, as analyzed by the same Sysmex Cell Counter NE8000 as mentioned above. Any specimen with abnormal or atypical leukocytes that had been re-analyzed by using another blood cell counter was excluded from the study. The differential percentages of the above five kinds of leukocytes add up to $100 \%$. The absolute count of a leukocyte subtype (in $10^{9} / \mathrm{L}$ ) was calculated as the product of its respective differential percentage and total leukocyte count.

Diagnostic criteria for hyperlipidemia. (i) Hypercholesterolemia: The diagnosis of normocholesterolemia was made when serum TC level was $<200 \mathrm{mg} / \mathrm{dL}$ and there was no history of treatment for hyperlipidemia. Untreated hypercholesterolemia was diagnosed in a subject with serum TC level $\geq 240 \mathrm{mg} / \mathrm{dL}$ and no previous treatment for hyperlipidemia. Untreated borderline hypercholesterolemia was diagnosed in a subject with serum TC level between 199 and $240 \mathrm{mg} / \mathrm{dL}$ and no previous treatment for hyperlipidemia. Those subjects with unknown or uncertain history of and/or treatment for hypercholesterolemia or who did not fit any of the above criteria for normocholesterolemia, untreated hypercholesterolemia, and untreated borderline hypercholesterolemia were classified as "others." (ii) Hypertriglyceridemia: The diagnosis of normotriglyceridemia was made when serum TG level was $<130 \mathrm{mg} / \mathrm{dL}$ and there was no history of treatment for hyperlipidemia. Untreated hypertriglyceridemia was diagnosed in a subject with serum TG level $\geq 200 \mathrm{mg} / \mathrm{dL}$ and no previous treatment for hyperlipidemia. Untreated borderline hypertriglyceridemia was diagnosed in a subject with serum TG level between 129 and $200 \mathrm{mg} / \mathrm{dL}$ and no previous treatment for hyperlipidemia. Those subjects with unknown or uncertain history of and/or treatment for hypertriglyceridemia or who did not fit any of above criteria for normotriglyceridemia, untreated hypertriglyceridemia, and untreated borderline hypertriglyceridemia were classified as "others." (iii) Pure or combined hyperlipidemia: In untreated hyperlipidemic subjects, the presence of hypercholesterolemia with normotriglyceridemia was diagnosed to have "pure hypercholesterolemia," and the presence of hypertriglyceridemia with normocholesterolemia was diagnosed to have "pure hypertriglyceridemia." Subjects with both hypercholesterolemia and hypertriglyceridemia were diagnosed as having "combined hyperlipidemia." Those who had hypercholesterolemia with borderline hypertriglyceridemia or had hypertriglyceridemia with borderline hypercholesterolemia were diagnosed as having "probable combined hyperlipidemia."

Statistical methods. The data in the study were analyzed by using the SAS statistical program (SAS, Cary, NC) as follows: (i) The following comparisons were analyzed by oneway analysis of variance (ANOVA) using Bonferroni method to compare pairs of means: various laboratory measurements in normolipidemic and different hyperlipidemic groups (Tables 1 and 2), and differential leukocyte counts in negative, borderline, and positive groups for different types of hyperlipidemia separately (Table 3); (ii) the following comparisons were analyzed by two-way ANOVA: differential leukocyte counts between nonsmokers and smokers adjusted for the presence and degree of hyperlipidemia (Table 4) and differential leukocyte counts by three levels of BMI adjusted for the presence and degree of hyperlipidemia (Table 5); and (iii) multivariate regression analyses of differential leukocyte counts on age, gender, BMI, smoking, and serum lipid levels in all study subjects $(n=3,282)$ were estimated by the ordinary least squares method (Tables 6 and 7).

\section{RESULTS}

Table 1 shows the results of comparisons of age, various serum lipid levels, and differential leukocyte counts in normolipidemic, untreated borderline hyperlipidemic, and untreated hyperlipidemic groups by one-way ANOVA. The serum levels of TC, LDL-cholesterol, and TG and the counts of all leukocytes, neutrophils, lymphocytes, and basophils all had a significantly positive association with the presence and degree of hyperlipidemia $(P<0.0001)$. The serum HDL-cholesterol level, on the contrary, had a significantly negative correlation with the presence and degree of hyperlipidemia $(P<0.0001)$. The monocyte count in the hyperlipidemic group, but not in the borderline hyperlipidemic group, was significantly higher than that in the normolipidemic group $(P<0.05)$. There was no significant difference in eosinophil count in these three groups.

The results of comparisons of various laboratory measure- 
TABLE 1

Comparisons of Age, Serum Lipid Levels, and Peripheral Differential Leukocyte Counts in Normolipidemic, Untreated Borderline Hyperlipidemic, and Untreated Hyperlipidemic Groups by One-way ANOVA ${ }^{a}$

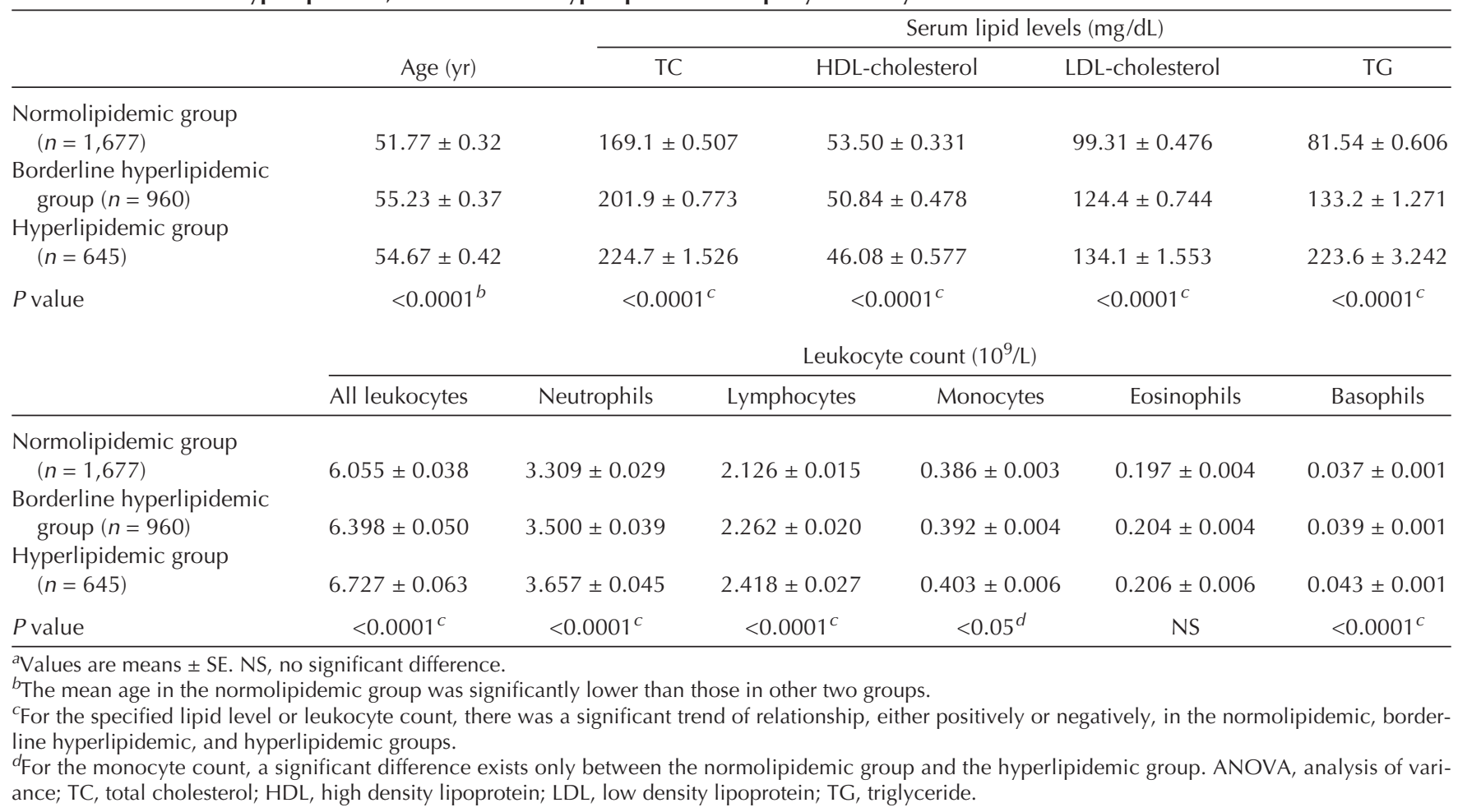

ments in normolipidemic, pure hypercholesterolemic, pure hypertriglyceridemic, combined hyperlipidemic, and probable combined hyperlipidemic groups by one-way ANOVA are shown in Table 2. Major findings include the following: (i) In the pure hypertriglyceridemic group, the total leukocyte count and counts of all leukocyte subtypes were significantly higher than those in the normolipidemic group. (ii) In contrast, in the pure hypercholesterolemic group, the total leukocyte count and counts of all leukocyte subtypes were not significantly different from those in the normolipidemic group. The monocyte count in the pure hypercholesterolemic group was significantly lower than that in normolipidemic group when compared by Student's $t$ test $\left[(0.350 \pm 0.015) \times 10^{9} / \mathrm{L}, n=\right.$ 102 , and $(0.386 \pm 0.003) \times 10^{9} / \mathrm{L}, n=1,677$, respectively, $P<$ $0.05]$. (iii) In the combined and the probable combined hyperlipidemic groups, the total leukocyte count and counts of neutrophils, lymphocytes, and basophils were significantly higher than those in the normolipidemic group. The obviously lower monocyte count in the patients with pure hypercholesterolemia was not seen in these combined hyperlipidemic groups.

The results of comparisons of differential leukocyte counts in negative, borderline, and positive groups for hypercholesterolemia, pure hypercholesterolemia, hypertriglyceridemia, and pure hypertriglyceridemia, made separately by one-way ANOVA, are listed in Table 3. The results confirmed several interesting findings in Tables 1 and 2. (i) In both the hypertriglyceridemic and the pure hypertriglyceridemic groups, the total leukocyte count and counts of all leukocyte subtypes were significantly higher than those in the normolipidemic group and showed significantly positive trends of relationships with the presence and degree of hypertriglyceridemia. (ii) In contrast, in the pure hypercholesterolemic group, only the monocyte count was significantly different from that in the normolipidemic group. It showed a negative relationship with the presence and degree of hypercholesterolemia. The significantly lower monocyte count in the pure hypercholesterolemic group, as compared with the normolipidemic group, did not exist in the hypercholesterolemic group that contained subjects with hypertriglyceridemia.

Because of well-documented positive associations between smoking and lipid disorders $(14,15)$ and higher peripheral total leukocyte counts $(1,3,13,17,18)$, we compared differential leukocyte counts between smokers and nonsmokers in normolipidemic, borderline hyperlipidemic, and hyperlipidemic groups by two-way ANOVA, as shown in Table 4. This analysis confirmed a higher total leukocyte count $(P<$ $0.0001)$, resulting from higher counts of all leukocyte subtypes ( $P$ values all $<0.0001$ ), in smokers after adjustments for presence and degree of hyperlipidemia. Obesity has been also associated with both lipid disorders (16) and higher peripheral total leukocyte counts $(18,19)$. Comparisons of differential leukocyte counts by three levels of BMI in normolipidemic, borderline hyperlipidemic, and hyperlipidemic groups by two-way ANOVA showed a significantly positive relationship between BMI and counts of all leukocytes, neutrophils, 
TABLE 2

Comparisons of Age, Serum Lipid Levels, and Peripheral Differential Leukocyte Counts Among Normolipidemic Group and Subgroups of Untreated Hyperlipidemia by One-way ANOVA ${ }^{a}$

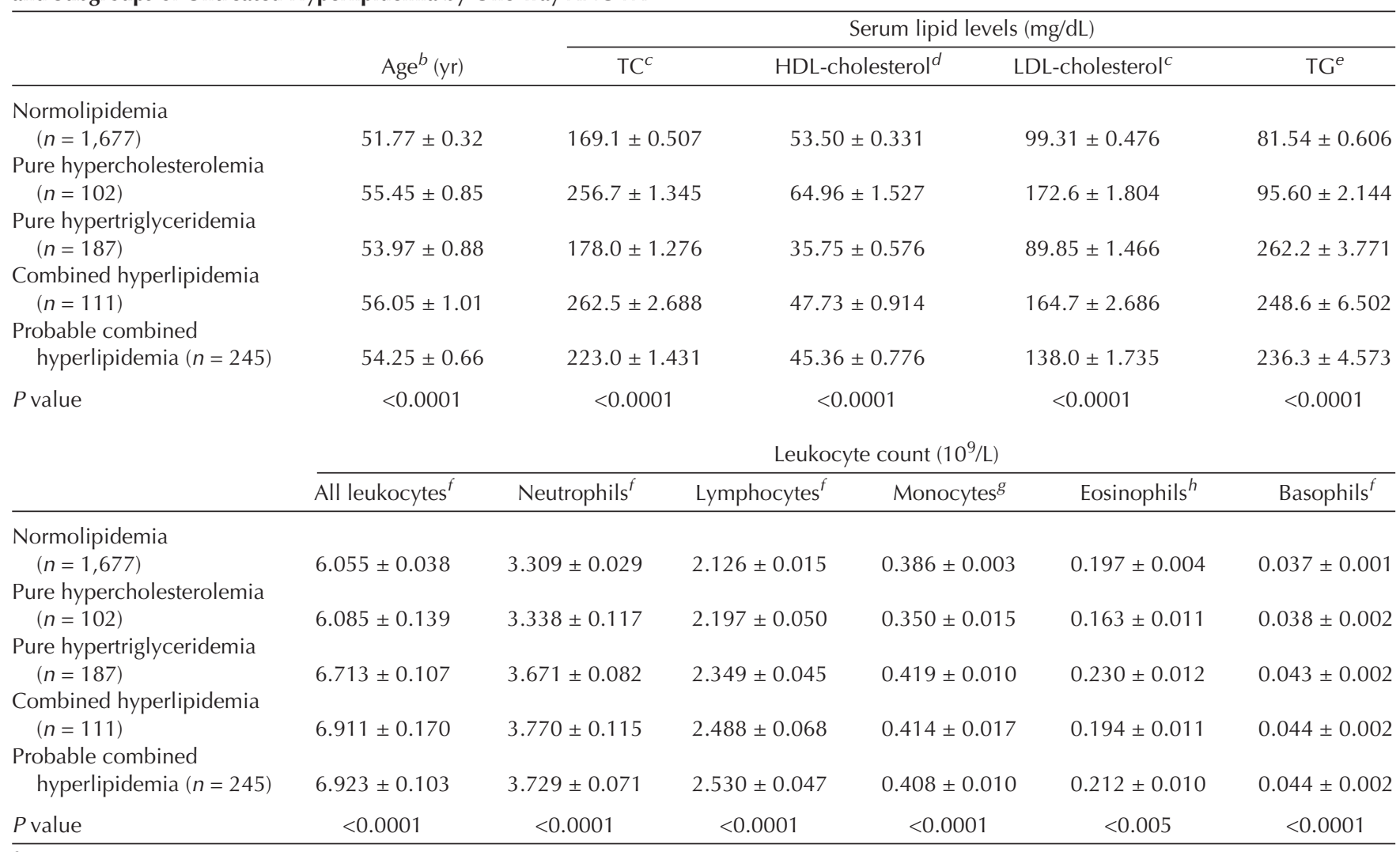

${ }^{a}$ Values are means $\pm \mathrm{SE}$.

${ }^{b}$ For age, significant difference exists only between the normolipidemic and the combined hyperlipidemic groups.

${ }^{c}$ For TC and LDL-cholesterol levels, there was no significant difference between the pure hypercholesterolemic and the combined hyperlipidemic groups.

${ }^{d}$ For HDL-cholesterol level, there was no significant difference between the combined hyperlipidemic and the probable combined hyperlipidemic groups.

eFor TG level, significant difference exists between any two study groups.

${ }^{f}$ For all leukocytes, neutrophils, lymphocytes, and basophils, the counts from the normolipidemic group were significantly lower than those in other study groups except for the pure hypercholesterolemic group.

${ }^{g}$ For monocytes, the count for the normolipidemic group was only significantly lower than that in the pure hypertriglyceridemic group, and the pure hypercholesterolemic group was significantly lower than those in other three hyperlipidemic groups.


cholesterolemic group was significantly lower than those in pure hypertriglyceridemic and probable combined hyperlipidemic groups. For abbreviations see Table 1.

lymphocytes, and monocytes after adjustments for presence and degree of hyperlipidemia (Table 5).

In all subjects taken together $(n=3,282)$, there were universal significant linear correlations between any two levels of serum TC, HDL-cholesterol, LDL-cholesterol, and TG. The correlation coefficients and probability values estimated by Pearson's analysis were as follows (data not shown in tables): TC and HDL-cholesterol, $R=0.1904, P<0.0001$; TC and LDL-cholesterol, $R=0.9041, P<0.0001$; TC and TG, $R=0.3473, P<0.0001$; HDL-cholesterol and LDL-cholesterol, $R=-0.0474, P<0.01$; HDL-cholesterol and TG, $R=$ $-0.4596, P<0.0001$; and LDL-cholesterol and TG, $R=$ $0.1570, P<0.0001$. These significantly linear correlations are likely to have a great influence on the demonstration of true correlations between each lipid level and the differential leukocyte counts in a multivariate regression analysis including all lipid levels. Therefore, we performed the multivariate regression analysis repeatedly using one kind of lipid level each time, adjusted for age, gender, BMI, and smoking. The results, as listed in Table 6, revealed the following: (i) Smoking was associated independently with higher total leukocyte count and counts of all leukocyte subtypes; (ii) age had independently negative correlations with total leukocyte count and counts of all leukocyte subtypes except neutrophils; (iii) BMI had independently positive correlations with total leukocyte count and counts of neutrophils, lymphocytes, and monocytes; (iv) men had significantly higher counts of monocytes and eosinophils; (v) serum levels of TC, LDL-cholesterol, and TG all had independently positive correlations with total leukocyte count $(P$ values all $<0.0001)$, neutrophil count $(P<0.01,<0.01$, and $<0.0001$, respectively), and lymphocyte count $(P$ values all $<0.0001)$; (vi) serum TC and TG levels also had independently positive correlations with basophil count $(P<0.01$ and $<0.0001$, respectively). Serum TC level 
TABLE 3

Comparisons of Differential Leukocyte Counts in Negative, Borderline, and Positive Groups of Different Hyperlipidemia Separately by One-way ANOVA ${ }^{a}$

\begin{tabular}{|c|c|c|c|c|c|c|}
\hline & \multicolumn{6}{|c|}{ Leukocyte count $\left(10^{9} / \mathrm{L}\right)$} \\
\hline & All leukocytes & Neutrophils & Lymphocytes & Monocytes & Eosinophils & Basophils \\
\hline \multicolumn{7}{|l|}{ Hypercholesterolemia $^{b}$} \\
\hline No $(n=1,677)$ & $6.055 \pm 0.038$ & $3.309 \pm 0.029$ & $2.126 \pm 0.015$ & $0.386 \pm 0.003$ & $0.197 \pm 0.004$ & $0.037 \pm 0.001$ \\
\hline Borderline $(n=789)$ & $6.511 \pm 0.057$ & $3.542 \pm 0.042$ & $2.323 \pm 0.024$ & $0.395 \pm 0.005$ & $0.211 \pm 0.005$ & $0.041 \pm 0.001$ \\
\hline Yes $(n=269)$ & $6.536 \pm 0.097$ & $3.567 \pm 0.070$ & $2.364 \pm 0.039$ & $0.380 \pm 0.010$ & $0.183 \pm 0.007$ & $0.041 \pm 0.001$ \\
\hline$P$ value & $<0.0001^{c}$ & $<0.0001^{C}$ & $<0.0001^{c}$ & NS & $<0.05^{d}$ & $<0.0001^{d}$ \\
\hline \multicolumn{7}{|c|}{ Pure hypercholesterolemia ${ }^{b}$} \\
\hline No $(n=1,677)$ & $6.055 \pm 0.038$ & $3.309 \pm 0.029$ & $2.126 \pm 0.015$ & $0.386 \pm 0.003$ & $0.197 \pm 0.004$ & $0.037 \pm 0.001$ \\
\hline Borderline $(n=381)$ & $6.129 \pm 0.076$ & $3.323 \pm 0.059$ & $2.194 \pm 0.031$ & $0.370 \pm 0.007$ & $0.203 \pm 0.008$ & $0.038 \pm 0.001$ \\
\hline Yes $(n=102)$ & $6.085 \pm 0.139$ & $3.338 \pm 0.117$ & $2.197 \pm 0.050$ & $0.350 \pm 0.015$ & $0.163 \pm 0.011$ & $0.038 \pm 0.002$ \\
\hline$P$ value & NS & NS & NS & $<0.05^{d}$ & $<0.05^{d}$ & NS \\
\hline \multicolumn{7}{|l|}{ Hypertriglyceridemia $^{b}$} \\
\hline No $(n=1,677)$ & $6.055 \pm 0.038$ & $3.309 \pm 0.029$ & $2.126 \pm 0.015$ & $0.386 \pm 0.003$ & $0.197 \pm 0.004$ & $0.037 \pm 0.001$ \\
\hline Borderline $(n=366)$ & $6.550 \pm 0.076$ & $3.609 \pm 0.059$ & $2.307 \pm 0.031$ & $0.393 \pm 0.007$ & $0.201 \pm 0.006$ & $0.040 \pm 0.001$ \\
\hline Yes $(n=487)$ & $6.875 \pm 0.074$ & $3.733 \pm 0.053$ & $2.463 \pm 0.032$ & $0.418 \pm 0.007$ & $0.216 \pm 0.007$ & $0.044 \pm 0.001$ \\
\hline$P$ value & $<0.0001^{c}$ & $<0.0001^{c}$ & $<0.0001^{c}$ & $<0.0001^{C}$ & $<0.05^{d}$ & $<0.0001^{C}$ \\
\hline \multicolumn{7}{|c|}{ Pure hypertriglyceridemia $^{b}$} \\
\hline No $(n=1,677)$ & $6.055 \pm 0.038$ & $3.309 \pm 0.029$ & $2.126 \pm 0.015$ & $0.386 \pm 0.003$ & $0.197 \pm 0.004$ & $0.037 \pm 0.001$ \\
\hline Borderline $(n=208)$ & $6.462 \pm 0.102$ & $3.552 \pm 0.080$ & $2.275 \pm 0.041$ & $0.398 \pm 0.010$ & $0.197 \pm 0.008$ & $0.040 \pm 0.002$ \\
\hline Yes $(n=187)$ & $6.713 \pm 0.107$ & $3.671 \pm 0.082$ & $2.349 \pm 0.045$ & $0.419 \pm 0.010$ & $0.230 \pm 0.012$ & $0.043 \pm 0.002$ \\
\hline$P$ value & $<0.0001^{c}$ & $<0.0001^{c}$ & $<0.0001^{c}$ & $<0.01^{d}$ & $<0.05^{d}$ & $<0.0005^{d}$ \\
\hline
\end{tabular}

Values are means $\pm \mathrm{SE}$.

${ }^{b}$ The diagnosis of hypercholesterolemia did not exclude coexisting hypertriglyceridemia or borderline hypertriglyceridemia, whereas a diagnosis of pure hypercholesterolemia was made only when serum TG level was normal. The difference between hypertriglyceridemia and pure hypertriglyceridemia is similarly defined.

${ }^{C}$ For the specified leukocyte count, there was a significant positive trend of relationship in the negative, borderline, and positive groups.

${ }^{d}$ Significant difference exists only between the group with the largest mean value and the group with the smallest mean value. For abbreviations see Table 1 .

had an independently negative correlation with monocyte count $(P<0.05)$; (vii) serum HDL-cholesterol level had independently negative correlations with total leukocyte count $(P<0.0001)$, neutrophil count $(P<0.0001)$, monocyte count $(P<0.0001)$, and basophil count $(P<0.05)$; and (viii) interestingly, there was no significant correlation between the studied lipid levels and eosinophil count in these multivariate regression analyses.

To see which lipid levels were most closely associated with each type of leukocyte, we performed the multivariate regression analyses (Table 6) a second time including the serum levels of HDL-cholesterol, LDL-cholesterol, and TG simultaneously. The serum TC level was excluded because of its extremely strong positive linear correlation with serum LDL-cholesterol level $(R=0.9041)$. The major findings, as shown in Table 7, are as follows: (i) Serum TG level was an independently positive determinant for total leukocyte count and for counts of all leukocyte subtypes except eosinophils.

TABLE 4

Comparisons of Differential Leukocyte Counts Between Smokers and Nonsmokers in Different Hyperlipidemic Groups by Two-way ANOVA ${ }^{a}$

\begin{tabular}{|c|c|c|c|c|c|c|}
\hline & \multicolumn{6}{|c|}{ Leukocyte count $\left(10^{9} / \mathrm{L}\right)$} \\
\hline & All leukocytes & Neutrophils & Lymphocytes & Monocytes & Eosinophils & Basophils \\
\hline \multicolumn{7}{|l|}{ Normolipidemic group } \\
\hline Nonsmokers $(n=1,287)$ & $5.890 \pm 0.040$ & $3.225 \pm 0.031$ & $2.069 \pm 0.016$ & $0.373 \pm 0.004$ & $0.187 \pm 0.004$ & $0.036 \pm 0.001$ \\
\hline Smokers $(n=390)$ & $6.598 \pm 0.089$ & $3.583 \pm 0.072$ & $2.317 \pm 0.036$ & $0.429 \pm 0.008$ & $0.229 \pm 0.008$ & $0.040 \pm 0.001$ \\
\hline \multicolumn{7}{|c|}{ Borderline hyperlipidemic group } \\
\hline Nonsmokers $(n=698)$ & $6.251 \pm 0.057$ & $3.423 \pm 0.043$ & $2.210 \pm 0.022$ & $0.381 \pm 0.005$ & $0.198 \pm 0.005$ & $0.038 \pm 0.001$ \\
\hline Smokers $(n=262)$ & $6.791 \pm 0.104$ & $3.704 \pm 0.085$ & $2.402 \pm 0.041$ & $0.422 \pm 0.009$ & $0.221 \pm 0.009$ & $0.042 \pm 0.001$ \\
\hline \multicolumn{7}{|l|}{ Hyperlipidemic group } \\
\hline Nonsmokers $(n=473)$ & $6.430 \pm 0.063$ & $3.519 \pm 0.048$ & $2.303 \pm 0.027$ & $0.379 \pm 0.007$ & $0.187 \pm 0.006$ & $0.041 \pm 0.001$ \\
\hline Smokers $(n=172)$ & $7.547 \pm 0.142$ & $4.037 \pm 0.102$ & $2.732 \pm 0.060$ & $0.468 \pm 0.014$ & $0.260 \pm 0.014$ & $0.048 \pm 0.002$ \\
\hline$P$ value for lipid group ${ }^{b}$ & $<0.0001$ & $<0.0001$ & $<0.0001$ & $<0.05$ & NS & $<0.0001$ \\
\hline$P$ value for smoking ${ }^{c}$ & $<0.0001$ & $<0.0001$ & $<0.0001$ & $<0.0001$ & $<0.0001$ & $<0.0001$ \\
\hline
\end{tabular}

${ }^{a}$ Values are means \pm SE.

${ }^{b}$ Probability values were tested for different lipid groups and adjusted for smoking groups.

CProbability values were tested for smoking groups and adjusted for different lipid groups. For abbreviations see Table 1. 
TABLE 5

Comparisons of Differential Leukocyte Counts by Three Levels of BMI in Different Hyperlipidemic Groups by Two-way ANOVA ${ }^{a}$

\begin{tabular}{|c|c|c|c|c|c|c|}
\hline & \multicolumn{6}{|c|}{ Leukocyte count $\left(10^{9} / \mathrm{L}\right)$} \\
\hline & All leukocytes & Neutrophils & Lymphocytes & Monocytes & Eosinophils & Basophils \\
\hline \multicolumn{7}{|l|}{ Normolipidemic group } \\
\hline $\mathrm{BMI}<22(n=605)$ & $5.855 \pm 0.061$ & $3.160 \pm 0.047$ & $2.095 \pm 0.025$ & $0.370 \pm 0.005$ & $0.193 \pm 0.006$ & $0.036 \pm 0.001$ \\
\hline $\mathrm{BMI}=22-25(n=615)$ & $6.035 \pm 0.059$ & $3.282 \pm 0.045$ & $2.132 \pm 0.024$ & $0.383 \pm 0.006$ & $0.201 \pm 0.006$ & $0.037 \pm 0.001$ \\
\hline $\mathrm{BMI}>25(n=457)$ & $6.346 \pm 0.077$ & $3.542 \pm 0.061$ & $2.159 \pm 0.030$ & $0.411 \pm 0.007$ & $0.197 \pm 0.007$ & $0.037 \pm 0.001$ \\
\hline \multicolumn{7}{|c|}{ Borderline hyperlipidemic group } \\
\hline $\mathrm{BMI}<22(n=178)$ & $6.117 \pm 0.123$ & $3.364 \pm 0.091$ & $2.155 \pm 0.047$ & $0.362 \pm 0.010$ & $0.199 \pm 0.012$ & $0.038 \pm 0.002$ \\
\hline $\mathrm{BMI}=22-25(n=363)$ & $6.309 \pm 0.076$ & $3.433 \pm 0.060$ & $2.258 \pm 0.031$ & $0.383 \pm 0.007$ & $0.197 \pm 0.007$ & $0.038 \pm 0.001$ \\
\hline $\mathrm{BMI}>25(n=419)$ & $6.595 \pm 0.078$ & $3.616 \pm 0.062$ & $2.312 \pm 0.031$ & $0.413 \pm 0.007$ & $0.213 \pm 0.006$ & $0.041 \pm 0.001$ \\
\hline \multicolumn{7}{|l|}{ Hyperlipidemic group } \\
\hline $\mathrm{BMI}<22(n=66)$ & $6.556 \pm 0.191$ & $3.545 \pm 0.127$ & $2.402 \pm 0.085$ & $0.383 \pm 0.019$ & $0.184 \pm 0.017$ & $0.043 \pm 0.003$ \\
\hline $\mathrm{BMI}=22-25(n=251)$ & $6.594 \pm 0.102$ & $3.604 \pm 0.076$ & $2.345 \pm 0.039$ & $0.392 \pm 0.010$ & $0.211 \pm 0.008$ & $0.042 \pm 0.002$ \\
\hline $\mathrm{BMI}>25(n=328)$ & $6.864 \pm 0.087$ & $3.721 \pm 0.062$ & $2.477 \pm 0.039$ & $0.415 \pm 0.009$ & $0.207 \pm 0.009$ & $0.043 \pm 0.001$ \\
\hline$P$ value for lipid group ${ }^{b}$ & $<0.0001$ & $<0.0001$ & $<0.0001$ & NS & NS & $<0.0001$ \\
\hline$P$ value for $B M I$ level ${ }^{C}$ & $<0.0001$ & $<0.0001$ & $<0.001$ & $<0.0001$ & NS & NS \\
\hline
\end{tabular}

${ }^{a}$ Values are means \pm SE. In the statistical analysis, the normolipidemic, borderline hyperlipidemic, and hyperlipidemic groups were represented digitally by 0,1 , and 2, respectively, and the groups of $\mathrm{BMI}<22, \mathrm{BMI}=22-25$, and $\mathrm{BMI}>25$ were represented by 0,1 , and 2 , respectively.

${ }^{b}$ Probability values were tested for different lipid groups and adjusted for BMI levels.

${ }^{c}$ Probability values tested for BMI levels and adjusted for different lipid groups. BMI, weight $(\mathrm{kg}) / \mathrm{height}^{2}\left(\mathrm{~m}^{2}\right)$; for other abbreviations see Table 1 .

It was the only independent determinant for basophil count among the studied lipid levels; (ii) serum HDL-cholesterol level was an independently negative determinant for total leukocyte count and for counts of neutrophils and monocytes, and it was an independently positive determinant for lymphocyte count; and (iii) serum LDL-cholesterol level had independently positive correlations with total leukocyte count and counts of neutrophils and lymphocytes. The same multivariate regression analyses in Table 7 were performed once again after replacing the serum LDL-cholesterol level with serum TC level. The results were very similar to those in Table 7, probably because of the strong positive linear correlation between LDL-cholesterol level and TC level.

Among the 67 subjects not included in the above analyses owing to a very high TG level ( $\geq 450 \mathrm{mg} / \mathrm{dL})$ that interfered with the laboratory calculation of serum LDL-cholesterol level, 33 patients had never received medication for hyperlipidemia before their health check in 1998. The mean \pm SE of age, serum lipid levels, and differential leukocyte counts in these 33 untreated extremely hypertriglyceridemic subjects were as follows: age, $49.73 \pm 2.06 \mathrm{yr}$; TC level, $220.1 \pm 6.598$ $\mathrm{mg} / \mathrm{dL}$; HDL-cholesterol level, $35.15 \pm 1.346 \mathrm{mg} / \mathrm{dL}$; TG level, $561.8 \pm 19.32 \mathrm{mg} / \mathrm{dL}$; total leukocyte count, $(7.391 \pm$ $0.330) \times 10^{9} / \mathrm{L}$; neutrophil count, $(4.134 \pm 0.269) \times 10^{9} / \mathrm{L}$; lymphocyte count, $(2.501 \pm 0.099) \times 10^{9} / \mathrm{L}$; monocyte count, $(0.458 \pm 0.031) \times 10^{9} / \mathrm{L}$; eosinophil count, $(0.247 \pm 0.027) \times$ $10^{9} / \mathrm{L}$; and basophil count, $(0.051 \pm 0.005) \times 10^{9} / \mathrm{L}$. The means of these leukocyte counts were all obviously higher than those in the normolipidemic group, the untreated borderline hyperlipidemic group, and the untreated hyperlipidemic group (see data in Table 1), but probably because of a small sample size with a large SE in the extreme hypertriglyceridemic group, only the differences in total leukocyte count and counts of neutrophils and monocytes reach statistical signifi- cances estimated by using Student's $t$ test. When compared with the pure hypertriglyceridemic group (with serum TG level between 199 and $450 \mathrm{mg} / \mathrm{dL}$, see data in Table 2), the means of all leukocyte counts in the extreme hypertriglyceridemic group were also all higher, but only the differences in total leukocyte count and neutrophil count reach statistical significances by Student's $t$ test [ $6.713 \pm 0.107$ vs. $7.391 \pm$ $0.330) \times 10^{9} / \mathrm{L}$ and $(3.671 \pm 0.082$ vs. $4.134 \pm 0.269) \times 10^{9} / \mathrm{L}$, respectively, both $P<0.05]$.

\section{DISCUSSION}

Although several human studies have found different associations between peripheral total leukocyte count and different serum lipid levels (1-3), the actual relationships between counts of different leukocyte types and different patterns of hyperlipidemia in humans have been unclear. Our study has shown that different types of hyperlipidemia are associated with very different peripheral differential leukocyte counts (Tables 2 and 3). A higher peripheral total leukocyte count in human hyperlipidemia (Table 1) was associated with pure hypertriglyceridemia but not pure hypercholesterolemia (Table 2), and the higher total leukocyte count in hypertriglyceridemia seemed secondary to higher counts of all leukocyte subtypes (Tables 2 and 3). In multivariate regression analyses, we demonstrated that serum TG level had independent and positive correlations with total leukocyte count and counts of all leukocyte subtypes except eosinophils (Tables 6 and 7) and serum TG was the only lipid level having an independent correlation with basophil count (Table 7). The means of total leukocyte count and counts of all leukocyte subtypes in the extreme hypertriglyceridemic group were all higher than those in the pure hypertriglyceridemic group, although only the differences in total leukocyte count and neu- 
TABLE 6

Multivariate Regression Analyses of Differential Leukocyte Counts on Age, Gender, BMI, Smoking, and One of Serum Levels of TC, HDL-cholesterol, LDL-cholesterol, and TG in All Study Subjects $(n=3,282)$

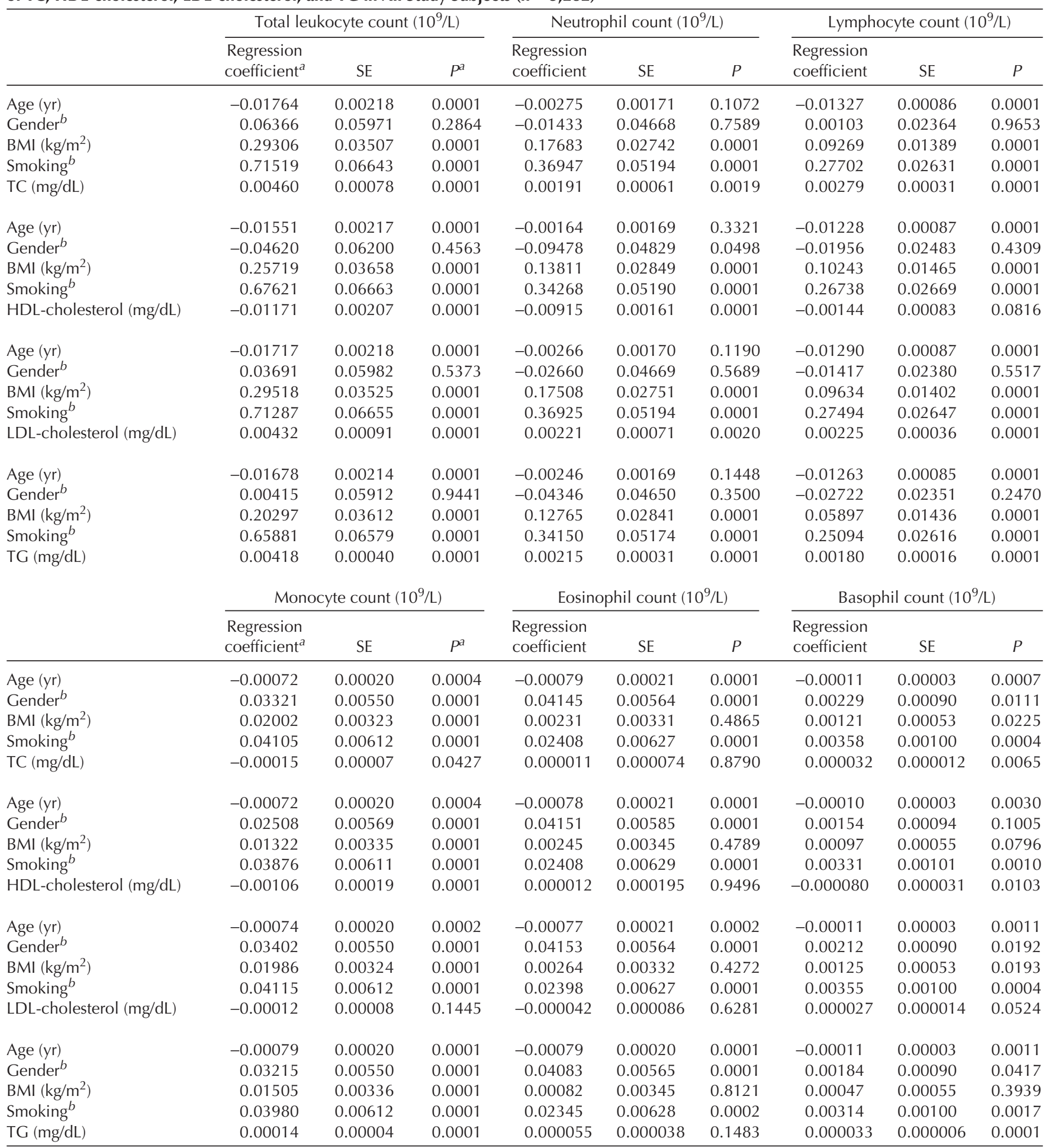

${ }^{a}$ Regression coefficients were estimated by ordinary least squares method, and probability values were calculated by $t$-test.

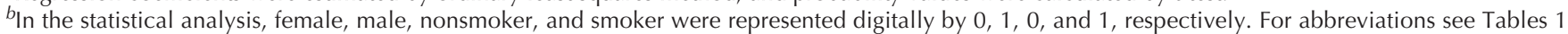
and 5.

trophil count reached statistical significances (see data in the last paragraph of the Results section). There is accumulating evidence from human studies that hypertriglyceridemia is a risk factor for atherosclerosis and CVD $(6,7)$, and it seems likely that different leukocytes play different roles in atherogenesis $(8-10,20)$. A higher peripheral total leukocyte count is associated with a higher risk of CVD (3,11-13) and the association of hypertriglyceridemia with a higher CVD risk 
TABLE 7

Multivariate Regression Analyses of Differential Leukocyte Counts on Age, Gender, BMI, Smoking, and Serum Levels of HDL-cholesterol, LDL-cholesterol, and TG in All Study Subjects $(n=3,282)$

\begin{tabular}{|c|c|c|c|c|c|c|c|c|c|}
\hline & \multicolumn{3}{|c|}{ Total leukocyte count $\left(10^{9} / \mathrm{L}\right)$} & \multicolumn{3}{|c|}{ Neutrophil count $\left(10^{9} / \mathrm{L}\right)$} & \multicolumn{3}{|c|}{ Lymphocyte count $\left(10^{9} / \mathrm{L}\right)$} \\
\hline & $\begin{array}{l}\text { Regression } \\
\text { coefficient }^{a}\end{array}$ & SE & $P^{a}$ & $\begin{array}{l}\text { Regression } \\
\text { coefficient }\end{array}$ & SE & $P$ & $\begin{array}{l}\text { Regression } \\
\text { coefficient }\end{array}$ & SE & $P$ \\
\hline Age (yr) & -0.01731 & 0.00215 & 0.0001 & -0.00252 & 0.00169 & 0.1368 & -0.01319 & 0.00085 & 0.0001 \\
\hline Gender $^{b}$ & -0.03993 & 0.06123 & 0.5144 & -0.09321 & 0.04816 & 0.0530 & -0.01633 & 0.02430 & 0.5016 \\
\hline BMI $\left(\mathrm{kg} / \mathrm{m}^{2}\right)$ & 0.16790 & 0.03719 & 0.0001 & 0.09639 & 0.02925 & 0.0010 & 0.05699 & 0.01476 & 0.0001 \\
\hline Smoking ${ }^{b}$ & 0.65873 & 0.06574 & 0.0001 & 0.33555 & 0.05171 & 0.0001 & 0.25845 & 0.02609 & 0.0001 \\
\hline HDL-cholesterol (mg/dL) & -0.00484 & 0.00220 & 0.0280 & -0.00614 & 0.00173 & 0.0004 & 0.00206 & 0.00087 & 0.0182 \\
\hline LDL-cholesterol (mg/dL) & 0.00358 & 0.00091 & 0.0001 & 0.00193 & 0.00071 & 0.0067 & 0.00181 & 0.00036 & 0.0001 \\
\hline TG $(\mathrm{mg} / \mathrm{dL})$ & 0.00368 & 0.00043 & 0.0001 & 0.00162 & 0.00034 & 0.0001 & 0.00188 & 0.00017 & 0.0001 \\
\hline
\end{tabular}

\begin{tabular}{|c|c|c|c|c|c|c|c|c|c|}
\hline & \multicolumn{3}{|c|}{ Monocyte count $\left(10^{9} / \mathrm{L}\right)$} & \multicolumn{3}{|c|}{ Eosinophil count $\left(10^{9} / \mathrm{L}\right)$} & \multicolumn{3}{|c|}{ Basophil count $\left(10^{9} / \mathrm{L}\right)$} \\
\hline & $\begin{array}{l}\text { Regression } \\
\text { coefficient }^{a}\end{array}$ & SE & $P^{a}$ & $\begin{array}{l}\text { Regression } \\
\text { coefficient }\end{array}$ & SE & $P$ & $\begin{array}{l}\text { Regression } \\
\text { coefficient }\end{array}$ & SE & $P$ \\
\hline Age $(y r)$ & -0.00070 & 0.00020 & 0.0005 & -0.00079 & 0.00021 & 0.0001 & -0.00011 & 0.00003 & 0.0008 \\
\hline Gender $^{b}$ & 0.02593 & 0.00569 & 0.0001 & 0.04204 & 0.00587 & 0.0001 & 0.00162 & 0.00094 & 0.0828 \\
\hline $\mathrm{BMI}\left(\mathrm{kg} / \mathrm{m}^{2}\right)$ & 0.01261 & 0.00346 & 0.0003 & 0.00163 & 0.00356 & 0.6475 & 0.00029 & 0.00057 & 0.6121 \\
\hline Smoking $b$ & 0.03802 & 0.00611 & 0.0001 & 0.02356 & 0.00630 & 0.0002 & 0.00315 & 0.00100 & 0.0017 \\
\hline HDL-cholesterol (mg/dL) & -0.00089 & 0.00020 & 0.0001 & 0.00015 & 0.00021 & 0.4868 & -0.000022 & 0.000034 & 0.5078 \\
\hline LDL-cholesterol (mg/dL) & -0.00013 & 0.00008 & 0.1243 & -0.000058 & 0.000087 & 0.5011 & 0.000020 & 0.000014 & 0.1414 \\
\hline TG (mg/dL) & 0.000083 & 0.000040 & 0.0373 & 0.000068 & 0.000041 & 0.0989 & 0.000031 & 0.000007 & 0.0001 \\
\hline
\end{tabular}

${ }^{a}$ Regression coefficients were estimated by ordinary least squares method, and probability values were calculated by $t$-test.

${ }^{b}$ In the statistical analysis, female, male, nonsmoker, and smoker were represented digitally by 0 , 1, 0, and 1, respectively. For abbreviations see Tables 1 and 5 .

may relate in part to its coexistence with higher peripheral counts of all leukocyte subtypes (Tables 2 and 3), probably except eosinophils (Tables 6 and 7).

For pure hypercholesterolemia, the results were quite different from those in pure hypertriglyceridemia. Compared with the normolipidemic group, pure hypercholesterolemia was associated with a significantly lower monocyte count and no significant difference in other leukocyte counts (Tables 2 and 3). Such a result may be partly related to the following complex relationships: (i) Both serum HDL-cholesterol and LDL-cholesterol levels were significantly higher in the pure hypercholesterolemic group (Table 2); (ii) there were independent and negative correlations between serum HDL-cholesterol level and total leukocyte count and counts of neutrophils and monocytes (Tables 6 and 7); and (iii) there were independent and positive correlations between serum LDLcholesterol level and total leukocyte count and counts of neutrophils and lymphocytes (Tables 6 and 7). A finding in Table 3 worth mentioning here is that the significantly lower monocyte count in the pure hypercholesterolemic group, as compared with the normolipidemic group, did not exist in the hypercholesterolemic group. The reason may be simply that the hypercholesterolemic group contained subjects with hypertriglyceridemia that was associated with a significantly higher monocyte count which had masked the presence of a significantly lower monocyte count associated with hypercholesterolemia.

The significantly lower monocyte count in pure hypercholesterolemia (Tables 2 and 3) and the independent and negative correlations between serum HDL-cholesterol level and counts of both monocytes and neutrophils (Tables 6 and 7) may be related to an anti-inflammatory effect of HDL particles. Several studies of humans have shown the abilities of HDL to bind inflammatory mediators, neutralize their leukocyte chemotactic activities, and thus attenuate the inflammation- or infection-induced increase in the count or recruitment of leukocytes, especially the monocytes and neutrophils (21, 22). In this way, HDL may ultimately reduce the peripheral monocyte and neutrophil counts. This may be one of the reasons that a high circulating HDL-cholesterol level is antiatherogenic $(23,24)$ in view of the possible underlying immunoinflammatory nature of atherosclerosis $(8,10)$.

Smoking has been well documented to have a positive effect on peripheral total leukocyte count $(17,18)$. Our study not only confirms such an independent and positive link between smoking and total leukocyte count but also reveals that such a link is related to independent and positive links between smoking and counts of all leukocyte subtypes (Tables 4, 6, and 7). These results strongly indicate that whenever peripheral leukocyte counts are the subjects of study, the factor of smoking should always be considered and included. Obesity has also been found to be associated with a higher peripheral total leukocyte count $(18,19)$. Our study confirms the existence of an independent and positive correlation between BMI and total leukocyte count and, furthermore, demonstrates independent and positive correlations between BMI and counts of neutrophils, lymphocytes, and monocytes (Tables 5, 6, and 7). These findings, together with other related results in the study and a positive association between obesity and lipid disorders (16), imply a close and complex inter- 
relationship among obesity, hyperlipidemia, and counts of neutrophils and monocytes.

In summary, our study revealed that an increase in peripheral total leukocyte count in hyperlipidemia is mainly associated with a higher serum TG level and the presence of hypertriglyceridemia. The higher total leukocyte count in hypertriglyceridemia is apparently due to increases in counts of all leukocyte subtypes except eosinophils. In contrast, pure hypercholesterolemia is associated with a lower monocyte count, possibly related to both a higher serum HDL-cholesterol level in pure hypercholesterolemia and a negative link between serum HDL-cholesterol level and monocyte count. Our study also reveals that smoking is independently associated with higher total leukocyte count and counts of all leukocyte subtypes, and BMI has independent and positive correlations with total leukocyte count and counts of neutrophils, monocytes, and lymphocytes.

\section{REFERENCES}

1. Hansen, L.K., Grimm, R.H., Jr., and Neaton, J.D. (1990) The Relationship of White Blood Cell Count to Other Cardiovascular Risk Factors, Int. J. Epidemiol. 19, 881-888.

2. Facchini, F., Hollenbeck, C.B., Chen, Y.N., Chen, Y.D., and Reaven, G.M. (1992) Demonstration of a Relationship Between White Blood Cell Count, Insulin Resistance, and Several Risk Factors for Coronary Heart Disease in Women, J. Intern. Med. 232, 267-272.

3. Nieto, F.J., Szklo, M., Folsom, A.R., Rock, R., and Mercuri, M. (1992) Leukocyte Count Correlates in Middle-aged Adults: The Atherosclerosis Risk in Communities (ARIC) Study, Am. J. Epidemiol. 136, 525-537.

4. Huang, Z.S., Wang, C.H., Yip, P.K., Yang, C.Y., and Lee, T.K. (1996) In Hypercholesterolemia, Lower Peripheral Monocyte Count Was Unique Among the Major Predictors of Atherosclerosis, Arterioscler. Thromb. Vasc. Biol. 16, 256-261.

5. Kannel, W.B., Castelli, W.P., and Gordon, T. (1979) Cholesterol in the Prediction of Atherosclerotic Disease. New Perspectives Based on the Framingham Study, Ann. Intern. Med. 90, 85-91.

6. Geurian, K., Pinson, J.B., and Weart, C.W. (1992) The Triglyceride Connection in Atherosclerosis, Ann. Pharmacother. 26, 1109-1117.

7. Postiglione, A., and Napoli, C. (1995) Hyperlipidaemia and Atherosclerotic Cerebrovascular Disease, Curr. Opin. Lipidol. 6, 236-242.

8. Kishikawa, H., Shimokama, T., and Watanabe, T. (1993) Localization of T Lymphocytes and Macrophages Expressing IL-1, IL-2 Receptor, IL-6 and TNF in Human Aortic Intima. Role of Cell-Mediated Immunity in Human Atherogenesis, Virchows Arch. 423, 433-442.
9. Blann, A.D., Seigneur, M., Adams, R.A., and McCollum, C.N. (1996) Neutrophil Elastase, von Willebrand Factor, Soluble Thrombomodulin and Percutaneous Oxygen in Peripheral Atherosclerosis, Eur. J. Vasc. Endovasc. Surg. 12, 218-222.

10. Ross, R. (1995) Cell Biology of Atherosclerosis, Annu. Rev. Physiol. 57, 791-804.

11. Prentice, R.L., Szatrowski, T.P., Fujikura, T., Kato, H., Mason, M.W., and Hamilton, H.H. (1982) Leukocyte Counts and Coronary Heart Disease in a Japanese Cohort, Am. J. Epidemiol. 116, 496-509.

12. Friedman, G.D., Tekawa, I., Grimm, R.H., Manolio, T., Shannon, S.G., and Sidney, S. (1990) The Leukocyte Count: Correlates and Relationship to Coronary Risk Factors (the CARDIA Study), Int. J. Epidemiol. 19, 889-893.

13. Kannel, W.B., Anderson, K., and Wilson, P.W. (1992) White Blood Cell Count and Cardiovascular Disease. Insights from the Framingham Study, JAMA 267, 1253-1256.

14. Mikhailidis, D.P., Papadakis, J.A., and Ganotakis, E.S. (1998) Smoking, Diabetes and Hyperlipidaemia, J.R. Soc. Health 118, 91-93.

15. Glueck, C.J., Heiss, G., Morrison, J.A., Khoury, P., and Moore, M. (1981) Alcohol Intake, Cigarette Smoking and Plasma Lipids and Lipoproteins in 12-19-Year-Old Children. The Collaborative Lipid Research Clinics Prevalence Study, Circulation 64, (3 Pt. 2):III, 48-56.

16. Despres, J.P. (1994) Dyslipidaemia and Obesity, Baillieres Clin. Endocrinol. Metab. 8, 629-660.

17. Ernst, E. (1995) Haemorheological Consequences of Chronic Cigarette Smoking, J. Cardiovasc. Risk. 2, 435-439.

18. Schwartz, J., and Weiss, S.T. (1991) Host and Environmental Factors Influencing the Peripheral Blood Leukocyte Count, Am. J. Epidemiol. 134, 1402-1409.

19. Pratley, R.E., Wilson, C., and Bogardus, C. (1995) Relation of the White Blood Cell Count to Obesity and Insulin Resistance: Effect of Race and Gender, Obesity Res. 3, 563-571.

20. Stuart, J., George, A.J., Davies, A.J., Aukland, A., and Hurlow, R.A. (1981) Haematological Stress Syndrome in Atherosclerosis, J. Clin. Pathol. 34, 464-467.

21. Badolato, R., Wang, J.M., Murphy, W.J., Lloyd, A.R., Michiel, D.F., Bausserman, L.L., Kelvin, D.J., and Oppenheim, J.J. (1994) Serum Amyloid A Is a Chemoattractant: Induction of Migration, Adhesion, and Tissue Infiltration of Monocytes and Polymorphonuclear Leukocytes, J. Exp. Med. 180, 203-209.

22. Pajkrt, D., Doran, J.E., Koster, F., Lerch, P.G., Arnet, B., van der Poll, T., ten Cate, J.W., and van Deventer, S.J. (1996) Antiinflammatory Effects of Reconstituted High-density Lipoprotein During Human Endotoxemia, J. Exp. Med. 184, 1601-1608.

23. Assmann, G., and Funke, H. (1990) HDL Metabolism and Atherosclerosis, J. Cardiovasc. Pharmacol. 16, 15-20.

24. Stein, O., and Stein, Y. (1999) Atheroprotective Mechanisms of HDL, Atherosclerosis 144, 285-301.

[Received February 23, 2000, and in final revised form January 30, 2001; revision accepted February 10, 2001] 